\title{
Pupil mapping Exoplanet Coronagraphic Observer (PECO)
}

\author{
Olivier Guyon ${ }^{a, b}$, James R.P. Angel ${ }^{a}$, Dana Backman ${ }^{c}$, Ruslan Belikov ${ }^{c}$, Donald Gavel ${ }^{d}$, Amir \\ Giveon $^{e}$, Thomas Greene ${ }^{c}$, Jeremy Kasdin ${ }^{f}$, James Kasting ${ }^{g}$, Marie Levine ${ }^{e}$, Mark Marley ${ }^{c}$, \\ Michael Meyer ${ }^{a}$, Glenn Schneider ${ }^{a}$, Gene Serabyn $^{e}$, Stuart Shaklan ${ }^{e}$, Michael Shao ${ }^{e}$, Motohide \\ Tamura $^{h}$, Domenick Tenerelli ${ }^{i}$, Wesley $\operatorname{Traub}^{e}$, John $\operatorname{Trauger}^{e}$, Robert Vanderbei ${ }^{f}$, Robert A. \\ Woodruff ${ }^{i}$, Neville J. Woolf ${ }^{a}$, Jeffrey $\mathrm{Wynn}^{j}$ \\ ${ }^{a}$ Steward Observatory, The University of Arizona, 933 N. Cherry Ave., Tucson, AZ 87521, USA \\ ${ }^{b}$ Subaru Telescope, NAOJ, 650 N. A'ohoku Pl., Hilo, HI, 96720, USA; \\ ${ }^{c}$ Ames Research Center, Moffett Field, CA 94035, USA \\ ${ }^{d}$ University of California, Santa Cruz, USA \\ ${ }^{e}$ Jet Propulsion Laboratory, USA \\ fPrinceton University, Princeton, NJ 08544, USA \\ ${ }^{g}$ Pennsylvania State University, Dept of Astronomy \& Astrophysics, 525 Davey Laboratory, \\ University Park, PA 16802, USA \\ ${ }^{h}$ National Astronomical Observatory of Japan, Osawa 2-21-1, Mitaka, Tokyo 181-8588, Japan \\ ${ }^{i}$ Lockheed Martin Space Corporation, USA \\ ${ }^{j}$ ITT Industries, USA
}

\begin{abstract}
The Pupil mapping Exoplanet Coronagraphic Observer (PECO) mission concept is a 1.4-m telescope aimed at imaging and characterizing extra-solar planetary systems at optical wavelengths. The coronagraphic method employed, Phase-Induced Amplitude Apodization or PIAA (a.k.a. pupil mapping) can deliver 1e-10 contrast at 2 lambda/D and uses almost all the starlight that passes through the aperture to maintain higher throughput and higher angular resolution than any other coronagraph or nuller, making PECO the theoretically most efficient existing approach for imaging extra-solar planetary systems. PECO's instrument also incorporates deformable mirrors for high accuracy wavefront control. Our studies show that a probe-scale PECO mission with $1.4 \mathrm{~m}$ aperture is extremely powerful, with the capability of imaging at spectral resolution $\mathrm{R} \approx 15$ the habitable zones of already known F, G, K stars with sensitivity sufficient to detect planets down to Earth size, and to map dust clouds down to a fraction of our zodiacal cloud dust brightness. PECO will acquire narrow field images simultaneously in 10 to 20 spectral bands covering wavelengths from 0.4 to $1.0 \mu \mathrm{m}$ and will utilize all available photons for maximum wavefront sensing and imaging/spectroscopy sensitivity. This approach is well suited for low-resolution spectral characterization of both planets and dust clouds with a moderately sized telescope.

We also report on recent results obtained with the laboratory prototype of a coronagraphic low order wavefront sensor (CLOWFS) for PIAA coronagraph. The CLOWFS is a key part of PECO's design and will enable high contrast at the very small PECO inner working angle.
\end{abstract}

Keywords: Coronagraphy, Adaptive Optics, Space Telescopes, Exoplanets

\section{INTRODUCTION}

More than 300 Exoplanets have now been identified thanks to indirect detection techniques (radial velocity, transits, microlensing). Within a short time, these techniques will allow detection of planets as small as Earth around the most favorable targets. In parallel, techniques and instruments for direct imaging of exoplanets are under development. Ground-based near-IR "Extreme-AO" systems equipped with coronagraphs will complement

Further author information: (Send correspondence to Olivier Guyon.) Olivier Guyon.: E-mail: guyon@naoj.org, Telephone: 18089345901

Space Telescopes and Instrumentation 2008: Optical, Infrared, and Millimeter, edited by Jacobus M. Oschmann, Jr., Mattheus W. M. de Graauw, Howard A. MacEwen, Proc. of SPIE Vol. 7010, 70101Y, (2008) · 0277-786X/08/\$18 · doi: 10.1117/12.790080

Proc. of SPIE Vol. 7010 70101Y-1 


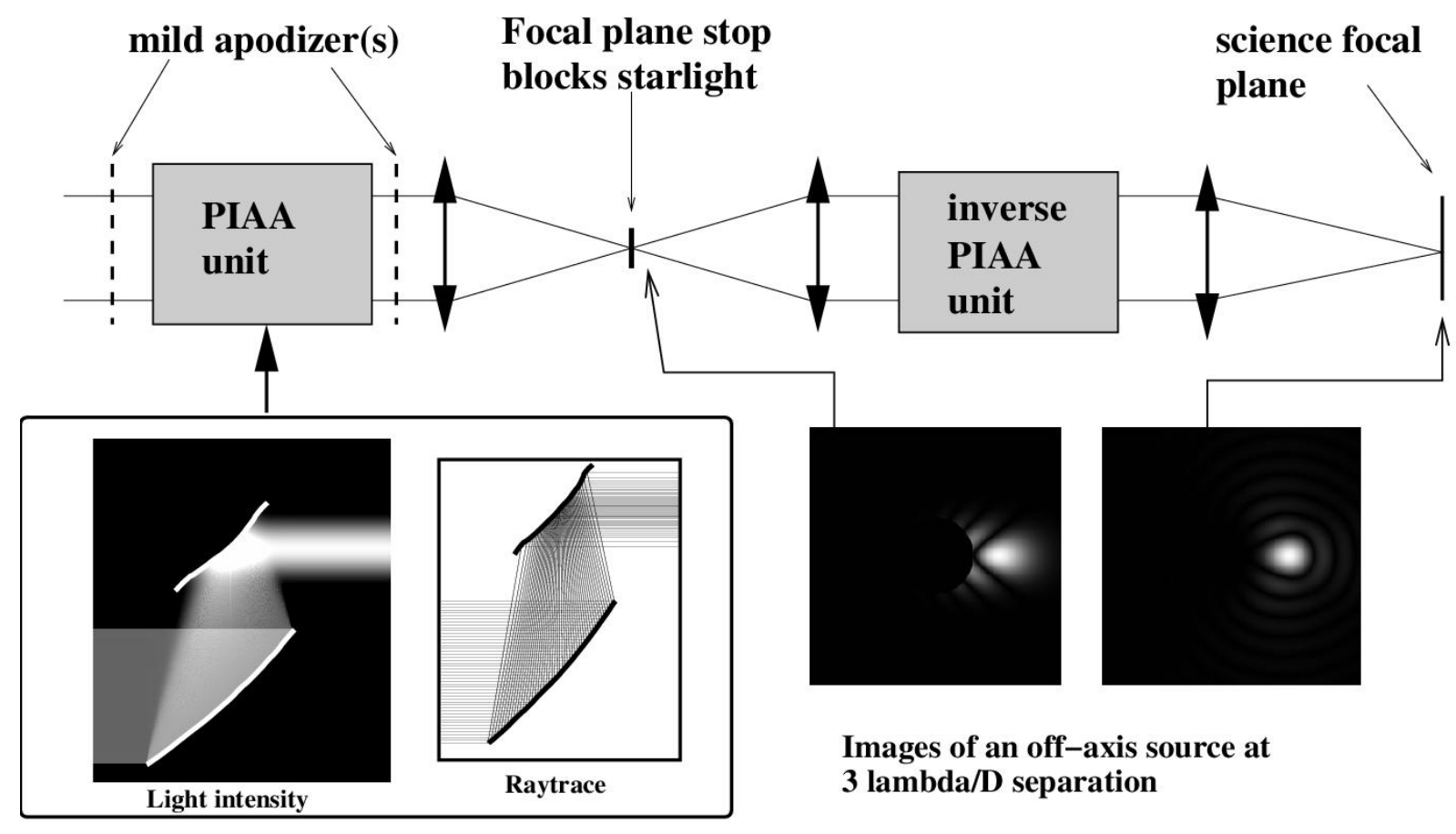

Figure 1. Schematic representation of the PIAAC. The telescope light beam enters from the left and is first apodized by the PIAA unit. Mild apodizer(s) are used to perform a small part of the apodization, and are essential to both mitigate chromatic diffraction propagation effects and allow for the design of "friendly" aspheric PIAA mirrors. An high contrast image is then formed, allowing starlight to be removed by a small focal plane occulting mask. An inverse PIAA unit is required to remove the off-axis aberrations introduced by the first set of PIAA optics.

indirect detection techniques by imaging young planetary systems (planets + dust) and detecting massive and young exoplanets at large (5 AU or more) distance from their host stars. Space coronagraphs working in the visible can directly image planets similar to the Earth around the most nearby stars. Imaging techniques also allow spectral characterization of exoplanets, with the ultimate goal of detecting "biomarkers" on habitable planets.

In this paper, we present a concept for an optical space telescope equipped with a coronagraph. PECO is sized at 1.4-m diameter to fit within the cost envelope of "probe-class" NASA missions. PECO uses a high performance PIAA coronagraph, described in $\S 2$, to allow direct imaging of planets as small as Earth around the most favorable stars. The overall PECO optical architecture, described in $\S 3$, is designed to maximize sensitivity for imaging and low-resolution spectroscopy of exoplanets, and makes optimal use of stellar photons for continuous wavefront sensing during observations. Thanks to its small inner working angle (IWA), PECO is particularly sensitive to low order aberrations such as pointing errors. We show in $\S 4$ a robust and efficient scheme to measure low order aberrations in the PECO coronagraph. Finally, in $\S 5$, we evaluate PECO's scientific capabilities for detection and characterization of exoplanets and dust.

\section{PHASE INDUCED AMPLITUDE APODIZATION (PIAA) CORONAGRAPH}

PECO uses a Phase-Induced Amplitude Apodization (PIAA) coronagraph to efficiently suppress stellar light. A detailed description of this coronagraph technique can be found in previous publications, ${ }^{1-8}$ and we briefly summarize in this section the principle and performance of PIAA coronagraphs.

As shown in Figure 1, in a PIAA coronagraph, the telescope beam is apodized by two aspheric mirrors which reshape the telescope beam. While a single aspheric mirror is sufficient to transform the top-hat illumination pattern of the telescope into a gaussian-like profile, the second mirror is necessary to re-collimate the output 
beam. The edges of an apodization profile suitable for high contrast imaging are very dark, and it would be very challenging to manufacture PIAA M1 mirror to project such a dark edge on PIAA M2: the surface curvature at the outer edge of PIAA M1 would need to vary rapidly over a small distance. The apodization is therefore shared between the aspheric mirrors (which perform most of the apodization) and conventional apodizer(s) which can be located before or after the PIAA mirrors. In addition to making PIAA M1 manufacturable, sharing the apodization with a conventional apodizer also greatly improves the chromaticity of the PIAA coronagraph. Thanks to the apodization, a high contrast image of the star is produced in the coronagraphic focal plane: a small occulting mask can therefore block starlight while having little effect on off-axis sources.

The beam shaping performed by the PIAA optics introduces strong off-axis aberrations which limit the useful field of view to about $8 \lambda / D$. The PIAAC shown in Figure 1 therefore includes an "inverse PIAA" to recover a wider unaberrated field of view in the science focal plane.

Thanks to the lossless apodization performed by the PIAA optics, the PECO coronagraph offers:

- High contrast. The PIAA optics and apodizer can be designed to produce very strong apodization to deliver $10^{-10}$ or higher contrast. The achievable contrast is in practice limited by wavefront quality. In a system which includes wavefront correction, as proposed for PECO, the wavefront quality is function of the wavefront control architecture (number, position and characteristics of deformable mirrors) and the wavefront stability (thermal variations, vibrations).

- Nearly $\mathbf{1 0 0 \%}$ throughput. In order to make PIAA M1 manufacturable, and to keep chromatic aberrations small, about $10 \%$ of the light is lost in the conventional apodizer(s). The PIAA system throughput is therefore about $90 \%$, without including losses in reflective coatings on the mirrors. This high throughput is essential for both science (exoplanets are faint) and wavefront control (minimizes the time necessary to measure wavefront aberrations).

- Small inner working angle. At $10^{-10}$ contrast, the PIAA inner working angle (IWA), defined as separation at which the throughput is $50 \%$ of its maximum, is slightly under $2 \lambda / D$. Designs with more aggressive IWA are possible, but they must overcome chromaticity issues and extreme sensitivity to low order aberrations and stellar angular diameter.

- Full 360 deg discovery space. High contrast can be achieved simultaneously in the full field of view with no "dead zone", as long as the wavefront control system architecture allows it.

- Full angular resolution. In the final science focal plane, the image of an off-axis source is only slightly $(\approx 5 \%)$ larger than in a conventional non-coronagraphic imaging telescope (the small difference is due to the conventional apodizer in the design). This ensures that a minimal amount of zodiacal and exozodiacal light is mixed with the planet's image, reduces risks of confusion between several planets, improves the astrometric precision for the planet's orbit, and provides sharp images of the exozodiacal cloud.

- Good achromaticity. With aspheric mirrors, the apodization is achieved by geometrical optics, and has very little dependance with wavelength. Diffraction propagation can however introduce chromatic effects if too much of the apodization is performed by the PIAA mirrors, or if the "sharing" of the apodization between the mirrors and the apodizer(s) is poorly chosen.

- Good tolerance to stellar angular diameter. Unlike other small-IWA $(<2 \lambda / d)$ coronagraphs, the PIAAC performance is only moderately affected by stellar angular size.

The PECO overall architecture, described in the next section, is aimed at fully exploiting these strengths.

\section{OVERALL ARCHITECTURE}

PECO is designed to make optimal use of incoming photons, with a large spectral coverage $(0.4 \mu \mathrm{m}$ to $1.0 \mu \mathrm{m})$. Maximizing the total number of photons transmitted to the detectors is essential for both science and wavefront control: 


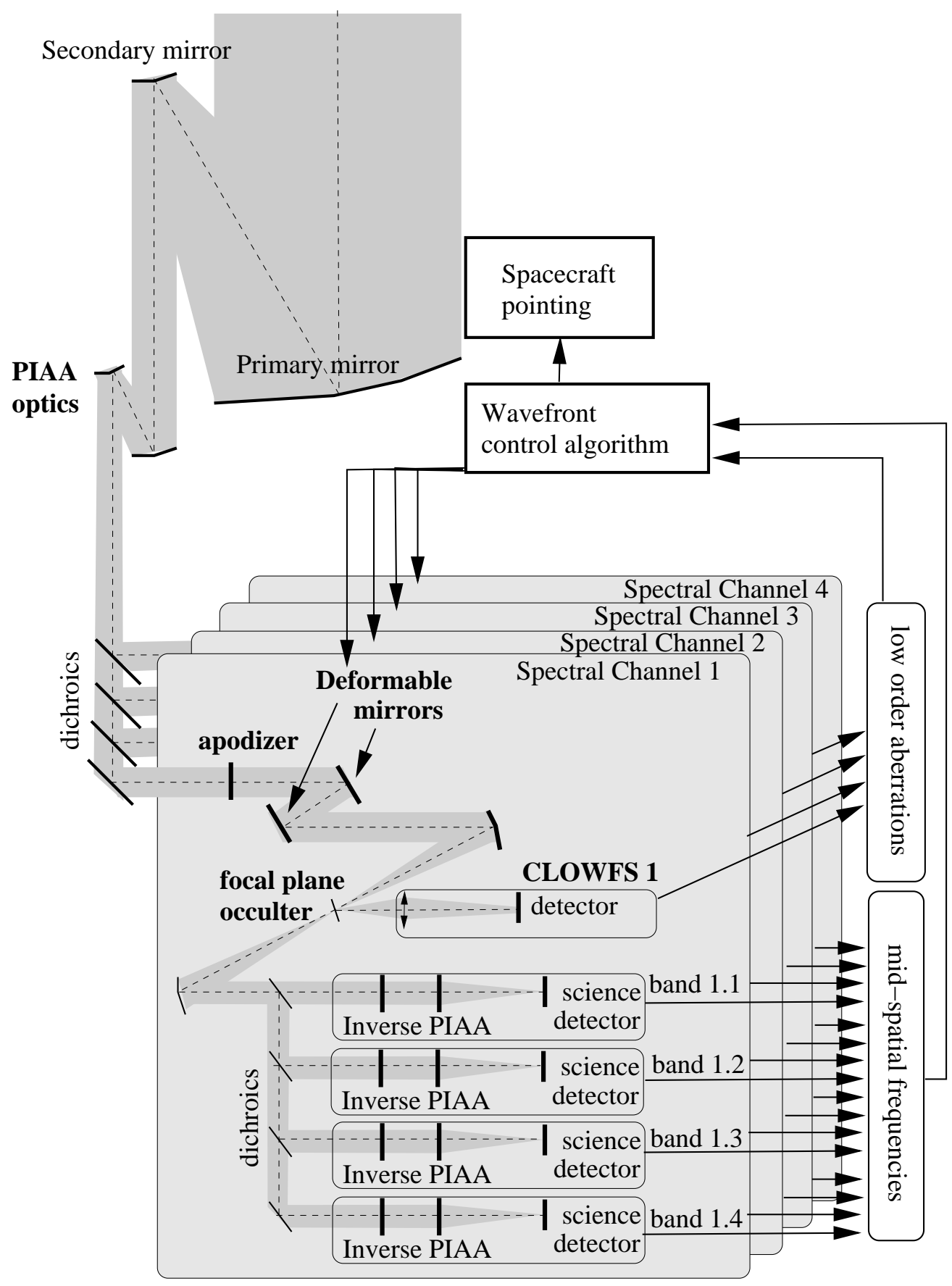

Figure 2. Conceptual representation of the PECO optical layout. The 1.4-m off-axis telescope's beam is apodized by the PIAA mirrors. A set of dichroics splits light in 4 spectral channels. The optical layout within one of these spectral channels is shown in this figure. See text for details. 
- Exoplanets are faint, and the total observing times required for detection with a 1.4-m telescope are long (typically a day per target) even in broadband.

- With more photons detected per unit of time, the wavefront control system is better able to track and correct aberrations.

Light however needs to be split into several wavelength channels for science (low resolution spectroscopy) and wavefront control (wavefront control can only be achieved over a limited spectral bandwidth due to slight chromatic variations in the wavefront). PECO therefore uses dichroics to split light in several wavelength channels to achieve the necessary spectral resolution without loosing light. While this results in a large number of components (optics, deformable mirrors, detectors), these components can be physically small thanks to the small field of view of PECO, and the system can tolerate lower performance of components after the dichroic, which would only compromise part of the spectral coverage.

A conceptual representation of the PECO architecture is shown in Figure 2.

PECO uses a 1.4-m diameter off-axis telescope. The surface accuracy of the primary and secondary mirrors does not exceed current manufacturing capabilities, as PECO's instrument includes wavefront sensing and correction to remove residual wavefront aberrations. A set of two aspheric PIAA mirrors apodizes the telescope beam.

Dichroics split the collimated output of the PIAA optics in 4 spectral channels, with a total spectral range from 0.4 to $1.0 \mu \mathrm{m}$. This split is necessary because both the wavefront control and PIAA coronagraph systems could not deliver the required performance over the full spectral range at once. In each spectral channel, an apodizer "completes" the apodization. Wavefront correction is achieved independently in each spectral channel with two deformable mirrors (DMs) in series to correct for both amplitude and phase errors and therefore take advantage of the $360 \mathrm{deg}$ high contrast field which the PIAA coronagraph can deliver. MEMS-type DMs are especially attractive for PECO: their small physical size $(\approx 10 \mathrm{~mm}$ across for $32 \times 32$ actuators $)$ is a good match to PECO's optical design, with a large number of spectral channels (4) and DMs (8 total). Larger Xinetics-type mirrors, which have already been used to demonstrate a contrast level close to PECO's requirement, ${ }^{10}$ could also be used with a larger beam size. A focal plane mask blocks starlight from the science path, and also serves as part of a high precision coronagraphic low order aberration wavefront sensor (CLOWFS), described in $\S 4$. The physical size of the focal plane mask is chosen to match the wavelength in each spectral channel (the mask is $\approx 2 \lambda / D$ in each channel, and is therefore physically larger in the red channel).

In each spectral channel, a second set of dichroics splits the light in 4 spectral bands to enable low resolution spectroscopy (with 16 spectral bands total). The exact number of spectral channels (currently baselined at 4) and spectral bands (currently baselined at 16) in PECO can be adjusted to meet science requirements and coronagraph/wavefront control performances.

Each spectral band includes a separate inverse PIAA to maintain high image quality over the full extent of the "dark hole" produced by the wavefront control system. Thanks to the small spectral coverage of each band and the location of the inverse PIAA systems (after the coronagraphic mask, where optical quality requirements are considerably relaxed), compact refractive optics can be used for the inverse PIAA.

Wavefront sensing is achieved by monitoring scattered light in the PECO science frames, which are acquired with photon-counting CCD read every few seconds. The DMs are continuously updated to correct for varying wavefront aberrations and provide the phase diversity signal necessary to measure the complex amplitude of starlight scattered by aberrations. ${ }^{11}$ Signals from all spectral channels can be added to improve wavefront sensing sensitivity in order to optimally track time-variable aberrations which are expected to be mostly achromatic.

\section{CORONAGRAPHIC LOW ORDER WAVEFRONT SENSOR}

High performance coronagraphs with small inner working angle (IWA) are unavoidably very sensitive to small pointing errors and other low order aberrations. This fundamental property is due to the fact that the wavefront of a source at small angular distance (typically between 1 and $2 \lambda / D$ ) from the optical axis is "similar" (in the linear algebra sense of the term) to an on-axis wavefront with a small $(<<\lambda / D)$ pointing error. Since all 


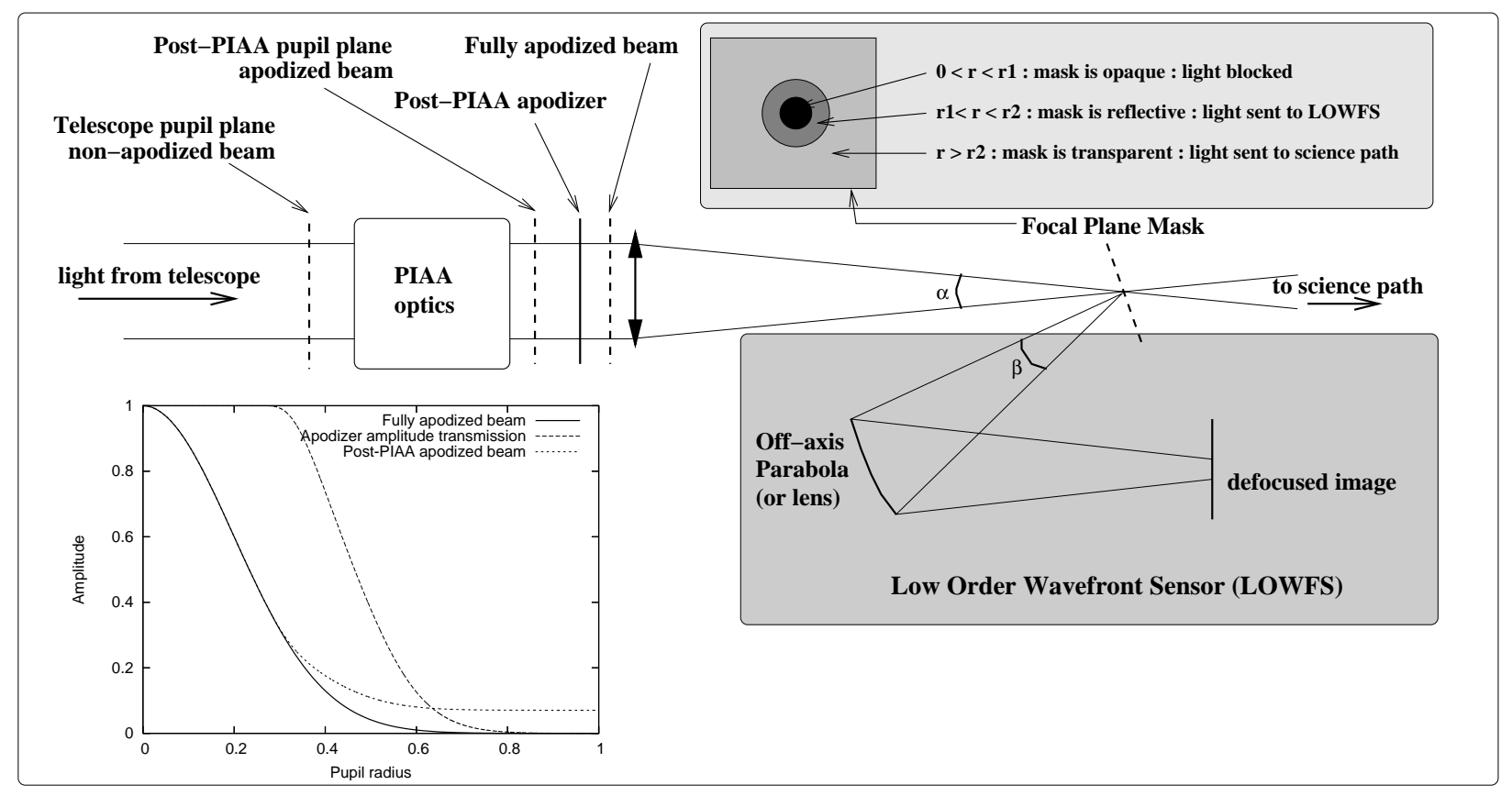

Figure 3. Optical layout of a coronagraphic low order wavefront sensor system for a PIAA coronagraph.

coronagraphs are linear systems in complex amplitude (the complex amplitude on every point of the science focal plane is a linear function of the 2-D complex amplitude in the input focal plane), if the coronagraph must "transmit" the former, it will also transmit a significant part of the latter, and therefore be extremely sensitive to pointing errors. This behavior is indeed verified by performance comparison between coronagraph concepts. ${ }^{9}$

Coronagraphic leaks produced by pointing errors will :

1. Add photon noise which will increase required PECO exposure times.

2. Produce signals which can be confused for a planet at or near the coronagraph's IWA. This effect can be compensated for if pointing errors are known.

3. Produce a coherent background which can constructively interfere with and amplify starlight speckles. This effect can also be compensated for if pointing errors are known.

Effects (2) and (3) are much more serious than (1): for a planet at $10^{-10}$ contrast, PECO can tolerate $\approx$ 0.4 mas of pointing error (similar to the angular radius of typical PECO target stars) before loosing SNR, but effects (2) and (3) require pointing errors to be known with an accuracy of 0.13 mas. A robust and accurate measurement of low order aberrations (especially tip-tilt errors, which are easily generated by telescope pointing errors and vibrations) is therefore essential for PECO.

In the coronagrapic low order wavefront sensor (CLOWFS) we plan to implement for PECO, light is extracted by the focal plane mask located after the PIAA optics to accurately measure pointing errors and other low order aberrations. In the PIAA coronagraph design, the role of this focal plane is to selectively remove starlight, while transmitting the science field. The mask is therefore illuminated by a large number of photons, which are freely available, and, if properly used, allow highly sensitive measurement of low order aberrations.

As shown in Figure 3, the CLOWFS produces a defocused image of a reflective focal plane ring to measure low order aberrations. Keeping the central part of the focal plane mask dark (non-reflective) amplifies the relative signal produced for a given pointing error and provides an ideally placed reference for the pointing measurement. Even for small levels of wavefront aberration, the proposed scheme produces large intensity signals which can 

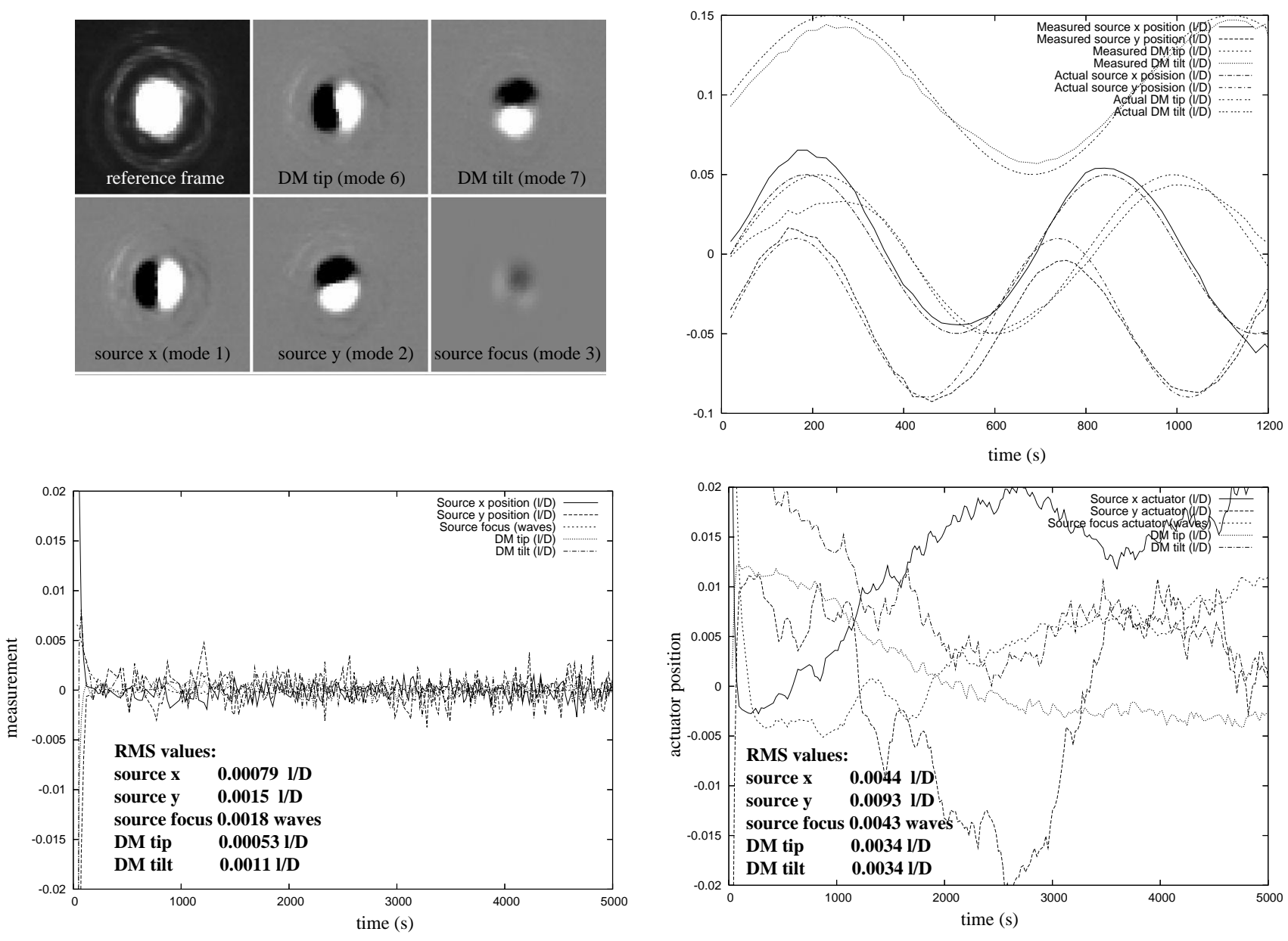

Figure 4. Laboratory performance for the CLOWFS. Upper left: Measured CLOWFS reference frame and influence functions for the 5 axis controlled in the experiment. Pre-PIAA and post-PIAA modes look extremely similar, as expected. Top right: Open loop simultaneous measurement of pre and post-PIAA modes. The measured amplitudes match very well the sine-wave signals sent to the actuators, and the CLOWFS is able to accurately measure all 4 modes shown here with little cross-talk. Since this measurement was performed in open loop, it also includes unknown drifts due to the limited stability of the testbed. Bottom left: Closed loop measurement of the residual error for the 5 modes controlled. The achieved pointing stability is about $10^{-3} \lambda / D$ for both the pre-PIAA and post-PIAA tip/tilt. Bottom right: Position of the actuators during the closed loop test.

be easily measured, and therefore does not require highly accurate calibration of either the detector or optical elements. The CLOWFS achieves nearly optimal sensitivity and is immune from non-common path errors. This technique is especially well suited for the PIAA coronagraph, and can unambiguously recover aberrations which originate from either side of the beam shaping introduced by the PIAA optics.

For small aberrations $(<1 \mathrm{rad})$, the CLOWFS image is a linear function of the low order aberrations in the system: each CLOWFS image is decomposed on a linear basis of modes to recover the values of tip, tilt, focus and astigmatism.

A prototype CLOWFS has been implemented in the PIAA coronagraph testbed at Subaru Telescope, and has already achieved the level of accuracy required for PECO. As shown in Figure $4, \mathrm{a} \approx 10^{-3} \lambda / D$ closed loop pointing stability has been achieved in the laboratory thanks to the CLOWFS. 


\section{EXOPLANET DETECTION AND CHARACTERIZATION WITH PECO}

The number of stars around which exoplanets can potentially be detected by PECO is listed in Table 1. For about 50 FGK main sequence stars, PECO could detect $(\mathrm{SNR}=5$ at $\mathrm{R}=5$ centered at $0.55 \mu \mathrm{m}$ ) within a day a planet in the habitable zone. PECO could detect an Earth analog around 20 stars. For most planet within the habitable zone, detection and spectral characterization are limited to the "blue" part of PECO's spectral coverage. PECO's observing strategy is to devote two out of the three years of the mission to the observation of the $\approx 50$ stars for which exoplanets could be imaged within the habitable zone. For this "deep" survey, planets will be most efficiently identified by PECO's blue spectral channels (better IWA) with several (10 or more) independent observations of the same target star.

PECO will perform higher SNR observation of the few most promising targets: Earth or Super-Earth exoplanets which have been identified in PECO's "deep survey" and/or have been known from other observations (for example ground-based radial velocity or space-based astrometry). For these few targets, PECO could accumulate up to several weeks of exposure time, scheduled when the planet separation is maximum to allow spectral characterization into the red end of PECO's spectral range. Since the location of these most attractive targets is presently unknown, it is difficult to predict at this time the SNR which would be achieved: an Earth-like planet around $\alpha$ Cent would be considerably easier to observe than a similar planet around the 20th easiest PECO target star. An Earth in the $\mathrm{HZ}$ of eps Eri would be imaged at $550 \mathrm{~nm}$ at R=5, SNR=20 in $24 \mathrm{hr}$ exposure in the presence of 1 zodi of exozodiacal dust.

Jupiter-like planets can be imaged by PECO around a significantly larger number of stars, and will be probed in both the deep survey and a larger but shallower survey of 150 additional targets. Many of the giant planets PECO will observe are already or will soon be known by ground-based radial velocity surveys. PECO can detect at least 12 of the known RV planets. For example, 47 Uma b would be detected in the $500 \mathrm{~nm}$ to $600 \mathrm{~nm}$ spectral range in just a few hours in the presence of 3 zodi of exozodi. PECO will acquire a statistically meaningful set of low resolution spectra for gas giants from Neptune mass to several times Jupiter mass.

Table 1. Number of FGK main sequence stars around which different planet types can be detected $(\mathrm{SNR}=5$ at $\mathrm{R}=5$ at 0.55 micron) with an ideal (perfect wavefront) PECO-sized (1.4-m) PIAA telescope, and with a smaller (1.0-m) and larger (1.8-m) PIAA telescope. Details of this simulation can be found in Guyon et al. 2006. This table assumes a 1 zodi cloud around each star and a 50\% throughput loss due to coatings and detector. The numbers given are for $20 \%$ detection probability for a single 1 day exposure with no prior information on the planet location, corresponding to $90 \%$ probability of at least one detection in 10 uncorrelated visits. Super Earths are assumed here to have 2x Earth radius. The HZ unit denotes the distance at which an Earth-like planet would have the same temperature as the Earth.

\begin{tabular}{|l|c|r|r|r|r|}
\hline & $\begin{array}{c}\text { Earth } \\
\text { at } 1 \mathrm{HZ} \\
\text { albedo } 0.3\end{array}$ & $\begin{array}{r}\text { SuperEarth } \\
\text { at } 1 \mathrm{HZ} \\
\text { albedo } 0.3\end{array}$ & $\begin{array}{r}\text { SuperEarth } \\
\text { at } 1.8 \mathrm{HZ} \\
\text { albedo } 0.3\end{array}$ & $\begin{array}{r}\text { Jupiter } \\
\text { at 1 AU } \\
\text { albedo 0.6 }\end{array}$ & $\begin{array}{r}\text { Jupiter } \\
\text { at 5 AU } \\
\text { albedo 0.6 }\end{array}$ \\
\hline 1.0-m PIAA & 5 & 13 & 23 & 21 & 437 \\
\hline 1.4 -m (PECO) & 20 & 38 & 56 & 52 & 1179 \\
\hline 1.8 -m PIAA & 41 & 79 & 127 & 103 & 2545 \\
\hline
\end{tabular}

PECO will also map with unprecedented sensitivity exozodial disks with $1 \lambda / D$ spatial resolution. Around the 50 "deep survey" targets, PECO will have the sensitivity to image exozodiacal fainter than our own. Simultaneous observation of planets and exozodiacal dust in many systems will greatly enhance our understanding of planetary systems architectures and evolution. The PECO debris disk and dust science objectives are:

1. Obtain a census of scattered light images of debris disks to the level of our own solar system and from habitable zones outwards

2. Observe the dynamical structures in disks to learn about the distribution of dust and any unseen planets

3. Characterize the physical properties of the debris dust in exoplanetary systems. 


\section{CONCLUSION}

Thanks to the use of a PIAA coroagraph with small IWA and high efficiency, PECO can image the habitable zones of nearby stars with sufficient sensitivity to acquire, on the most favorable targets, low resolution spectra of Earth-sized planets and, for a few tens of stars, map exozodiacal clouds down to sub-zodi levels. For a large number of giant planets, some of them already identified by radial velocity, PECO can acquire high SNR low resolution specta. The number of targets shown in Table 1 assumes a perfect wavefront and no overhead for wavefront control. An important part of our ongoing concept study is to quantify the effect, both in achievable contrast and wavefront sensing overhead, of the PECO wavefront control system. The full PECO optical train, including the telescope, must be kept very stable (with little vibrations and thermal disturbances) in order to allow the wavefront control system, which must work with a finite amount of photons from the star, to efficiently suppress diffracted light.

The PECO concept is scalable to larger telescope size. While we do not expect the instrument to become more challenging with increased telescope diameter, more spectral channels could be added to take advantage of higher SNR. With larger telescope diameter, the angular diameter of nearby stars will become too large to maintain a $2 \lambda / \mathrm{D}$ IWA at the $10^{-10}$ contrast level. Redesign of the PIAA coronagraph apodization profile and focal plane mask may therefore be necessary.

\section{ACKNOWLEDGMENTS}

Concept development for PECO is been supported by NASA under a Advance Mission Concept Study grant. Development of the PIAA coronagraph is supported by the National Astronomical Observatory of Japan, NASA Ames and NASA JPL.

\section{REFERENCES}

[1] Guyon, O. 2003, A\&A, 404, 379

[2] Traub, W.A., Vanderbei, R.J., 2003, ApJ, 599, 695

[3] Guyon, O., Pluzhnik, E.A., Galicher, R., Martinache, F., Ridgway, S.T., Woodruff, R.A. 2005, ApJ, 622,744

[4] Vanderbei, R.J., Traub, W.A. 2005, ApJ, 626, 1079

[5] Martinache, F., Guyon, O., Pluzhnik, E.A., Galicher, R., Ridgway, S.T. 2006, ApJ, 639, 1129

[6] Vanderbei, R.J. 2006, ApJ, 636, 528

[7] Pluzhnik, E.A., Guyon, O., Ridgway, S.T., Martinache, F., Woodruff, R.A., Blain, C., Galicher, R. 2006, ApJ, 644, 1246

[8] Pluzhnik, E.A., Guyon, O., Warren, M., Woodruff, R.A., Ridgway, S.T. 2006, SPIE 6265-135

[9] Guyon, O., Pluzhnik, E. A., Kuchner, M. J., Collins, B., Ridgway, S. T. 2066, ApJS, 167, 81

[10] Trauger, J.T., Traub, W.A. 2007, Nature, 446, 771

[11] Give'on, A., Kern, B., Shaklan, S., Moody, D.C. 2007, proc. SPIE vol 6691 